\title{
APLICAÇÃO DO BUSINESS PROCESS MANAGEMENT EM UMA CLÍNICA ODONTOLÓGICA UNIVERSITÁRIA
}

\section{APPLICATION OF BUSINESS PROCESS MANAGEMENT IN AN UNIVERSITY STUDENT DENTAL CLINIC}

\author{
Diego Silva Mota (Centro Universitário Católica de Quixadá - Unicatólica) \\ diego.silva.motta@gmail.com \\ Jair Paulino de Sales (Centro Universitário Católica de Quixadá - Unicatólica) \\ jairpsales@hotmail.com
}

\begin{abstract}
Resumo: Considerando a necessidade de implementação de um sistema de gerenciamento dos processos executados por uma clínica odontológica universitária, objetivando identificar e analisar os processos e fluxos internos que ocorrem em uma clínica odontológica de uma IES, propõe-se, por meio do modelo BPMN, um sistema de gerenciamento das atividades e etapas, mapeando-as. Para tanto, procede-se (i) o levantamento dos dados relacionados ao processo, (ii) mapeamento do processo e fluxo de atividades, (iii) aplicação de ferramentas da qualidade e por fim (iv) apresentação de um plano de ações composto por propostas de melhorias. Desse modo, observa-se que ao documentar o fluxo do processo com base na metodologia BPMN é possível melhorar significativamente seu desempenho, eliminando atividades que não agregam valor ao processo e padronizando a execução do mesmo. É possível concluir que podem ser obtidos ganhos significativos na eficiência do processo, impactando diretamente em uma melhor experiência aos usuários do mesmo, garantindo um aproveitamento melhor do tempo/disciplina por parte do aluno, bem como a satisfação do usuário enquanto comunidade local.
\end{abstract}

Palavras-chave: BMPN. Gestão de processos. Mapeamento.

Abstract: Considering the need to implement a system of processes management performed by a university dental clinic, aiming to identify and analyze the processes and inward flows that occur in a dental clinic of a HEI, it is proposed, through the BPMN model, a management system of activities and stages, mapping them. To this end, it is proceeded (i) the survey of data related to the process, (ii) process mapping and flow of activities, (iii) application of quality tools and finally (iv) presentation of an action plan consisting of proposals for improvements. Thus, it is observed that by documenting the process flow based on the BPMN methodology it is possible to significantly improve its performance, eliminating activities that do not add value to the process and standardizing its execution. It is possible to conclude that significant gains can be achieved in the process efficiency, directly impacting on a better experience for users, ensuring a better use of the student's time / discipline as well as user satisfaction while local community.

Keywords: BPMN. Mapping. Management of processes. 


\section{Introdução}

Advinda das inúmeras mudanças presentes ao longo da história e das escolas administrativas, a gestão baseada em processos abrange um conjunto de técnicas/metodologias que se aplicada continuamente, proporcionam melhorias significativas no desempenho dos processos e/ou organização como um todo (CARVALHO et al., 2005).

Essas melhorias, principalmente em tempos de crise, fazem-se necessárias para a diferenciação, enquanto organização, em relação a agregação de valor para o consumidor e deve estar relacionada às atividades que geram maior impacto nos quesitos fundamentais para a sobrevivência das organizações.

Para Slack et al (2013), a utilização de uma perspectiva de processos apresenta uma análise concisa da organização, seja ela produtora de bens ou serviços. Tal analise visa proporcionar o entendimento dos processos e operações realizados por cada um dos três níveis básicos: estratégico, tático e operacional.

Diante do contexto apresentado, compreende-se que uma empresa, seja ela pública ou privada, executa atividades e estas necessitam de processos bem formulados e otimizados que possibilitem agregar valor ao produto/serviço para o cliente. Não obstante, faz-se necessário também que a organização tenha em mente que o conjunto de todos os processos irá representala e que cada processo possui características exclusivas e particulares, que são imprescindíveis para o seu correto funcionamento e sobrevivência.

\section{Justificativa}

Tomando como base o atual estágio da sociedade, no que diz respeito ao desenvolvimento tecnológico, o aumento da competição e necessidade de adequação as constantes mudanças, pode-se afirmar que o gerenciamento de processos de negócios (BPM) se faz indispensável para uma organização, uma vez que as mesmas necessitam de um alto nível de controle em seus processos possibilitando automatiza-los com maior eficiência e eficácia além de atuar como facilitador na compreensão das necessidades advinda dos processos de negócio (KOSLOSKI, CALAZANS, \& GUIMARÃES, 2017).

Dessa forma, conforme exposto, verifica-se a grande importância da análise dos processos organizacionais, independentemente do tipo de empresa, seja ela prestadora de serviços e/ou produtora de bens. Portanto, diante da problemática existente relativa à a necessidade de 
implementação de um sistema de gerenciamento dos processos que ocorrem em uma clínica odontológica de uma Instituição de Ensino Superior (IES), o presente trabalho encontra sua justificativa na necessidade não somente de avaliar, mas também de propor medidas que viabilizem a execução acertada das atividades e o seu correto mapeamento. Para isso, propõese o uso do modelo Business Process Model and Notation (BPMN).

\section{Objetivo Geral}

Identificar e analisar os processos e fluxos internos que ocorrem em uma clínica odontológica de uma IES e propor, por meio do modelo $B P M N$, um sistema de gerenciamento das atividades e etapas, mapeando-as.

\subsection{Objetivos Específicos}

- Identificar os processos da clínica odontológica;

- Analisar os processos e verificar os pontos críticos intrínsecos às atividades que são executadas;

- Mapear e reorganizar os processos com base no modelo BPMN;

- Sugerir melhorias com base no modelo utilizado.

\section{Referencial Teórico}

Nesta secção será apresentada uma revisão teoria sobre os temas abordados neste trabalho.

\subsection{Gestão Baseada em Processos}

Segundo Paim (2009), a transição entre melhoria de operações e melhoria de processo partiu da ideia de divisão do trabalho, uma vez que proporcionou uma radical separação entre ambos. É válido ressalta que antes da divisão do trabalho, um único indivíduo era responsável pelo processo global de produção e com esse novo modelo, além de aumentos consideráveis na produtividade, surgiu também uma maior exigência de gerenciamento.

Neste contexto, o termo 'processo' era definido como um grande aglomerado de operações, onde a melhoria das mesmas de forma isolada, não significava necessariamente a melhoria do processo global de produção. O surgimento do Sistema Toyota de Produção rompeu o paradigma de melhorias de operações, o que provocou o direcionamento dos esforços de melhoria para os processos como um todo. Tal fato provou que era possível manter um alto 
nível de operação com um grande grau de flexibilidade e em larga escala, derrubando o modelo fordista e revolucionando a maneira de produzir.

Devido às necessidades organizacionais, tem-se verificado ao longo da história, uma preocupação crescente no que diz respeito à gestão das atividades que são desenvolvidas, bem como dos processos relacionados a elas.

Nesse contexto, com o advento da segunda revolução industrial e com as contribuições advindas do estudo de tempos e movimentos idealizado por Frederick Taylor, Pradella et al (2012) relatam que o conceito de gestão dessas atividades e/ou processos, passaram a ser o foco principal da administração moderna. Isso porque, com base no gerenciamento de processos, verificou-se a possibilidade de se alcançar melhorias quantitativas, além de permitir uma visão sistêmica e objetiva das atividades, estruturas e recursos. É válido ressaltar que esses devem estar alinhados aos objetivos organizacionais e devem promover uma maior fluidez em seus fluxos.

\subsection{Modelo BPM}

Para Brock (2013), a gestão de processos de negócio (BPM - Business Process Management) toma por base duas abordagens fundamentais, sendo a primeira desenvolvida por Shewhart e Deming (SHEWHART; DEMING, 1986; DEMING, 1953) e a segunda por Hammer e Champy (HAMMER, 1990; HAMMER; CHAMPY, 1993).

Especificamente, no primeiro trabalho, os autores deram origem ao atual modelo de qualidade (Seis Sigma), com base no estudo sobre controle estatístico de processos. Já no segundo baseiase no conceito de reengenharia de processos de negócio, que inseriu no estudo da administração conceitos como desenho, reorganização e modelagem dos processos de negócio.

No decorrer da última década, essas duas abordagens com perspectivas em melhoria de processos fundiram-se e deram origem à gestão de processos de negócio, que consiste em um sistema integrado de gestão de desempenho de negócios voltado para a gestão de processos de negócio ponta a ponta.

Segundo o BPM CBOK (2013), essa nova visão gerencial representa todo o trabalho executado na agregação de valor de um produto ou processo, independentemente de quais sejam os setores ou áreas organizacionais envolvidas, representando uma visualização que vai além das operações de negócio, começando em um nível mais alto do que o nível que de fato realiza a 
execução da tarefa e atuando com base na hierarquia dos processos permitindo o estabelecimento de princípios e práticas que garantem a eficiência organizacional na execução de seus processos de negócio.

\subsection{Ferramentas da Qualidade}

As ferramentas da qualidade, ou ferramentas administrativas, são um conjunto de técnicas utilizadas para o desenvolvimento de uma gestão empresarial centrada em promover um controle acertado de seus processos, métricas e objetivos. Ao tratar dos problemas administrativos Deming e Ishikawa afirmaram que cerca de $94 \%$ de todos os problemas são provenientes de causa comuns nos processos e métodos, dos quais $6 \%$ podem ser atribuídas ao nível operacional e que grande parte dos problemas enfrentados por uma organização são de responsabilidade da gerencia (MEIRELES, 2001).

Existem na literatura inúmeras ferramentas de controle, diagnóstico e melhoria de processos, das quais fazem parte as ferramentas da qualidade que são, por sua vez um conjunto de ferramentas complementares utilizadas para realização de diagnostico, identificação de causa, propostas de melhoria, controle de qualidade. Com o intuito de tornar este estudo mais objetivo, serão apresentados, a seguir apenas as ferramentas que possuem relevância ao desenvolvimento do mesmo, começando pelo Gráfico de Pareto, que, segundo Lins (1993) é utilizado na identificação das principais falhas de um processo, visando ataca-las efetivamente, obtendo assim o máximo de ganho no que se diz respeito a solução de problemas, está ferramenta se baseia em um princípio denominado de " $80 / 20 "$ no qual se afirma que $20 \%$ dos problemas, correspondem a $80 \%$ das causa. No entanto, existem inúmeras variações desta ferramenta a depender de sua necessidade de aplicação.

Uma vez identificada a falha principal, pode-se aplicar o diagrama de Ishikawa, ou diagrama de causa e feito, sendo este uma ferramenta de diagnóstico onde os colaboradores, independentemente de sua função dentro da empresa, podem contribuir para identificação das causas raízes de um determinado problema, ou da falha principal (BARRETO; LOPES, 2014). Segundo De Lima Junior e De Freitas (2005), atendendo a sequência lógica do processo de proposição de soluções, uma vez já identificada a falha principal e suas causas é chegado o momento da aplicação do 5W2H que consiste em reunir todo o staff da empresa, com objetivo de elaborar um quadro de soluções para uma determinada causa/problema pelos próprios stakeholders. 
Quadro 1 - Estruturação do 5W2H

\begin{tabular}{|c|c|c|}
\hline $5 \mathbf{W} 2 \mathrm{H}$ & Tradução & Perguntas estimuladas \\
\hline What? & O que? & O que é isso? O que aconteceu? O que pode ser feito? \\
\hline Who? & Quem? & Quem fez? Quem é responsável? A quem interessa? \\
\hline When? & Quando? & Quando aconteceu? Quando deve ser feito? Quanto tempo leva para fazer? \\
\hline Why? & Por quê? & Por que acontece? Por que isso deve ser feito? Quanto tempo leva para fazer? \\
\hline Where? & Onde? & Por que acontece? Porque isso deve ser feito? Quanto tempo leva para fazer? \\
\hline How? & Como? & Como será feito? \\
\hline $\begin{array}{c}\text { How } \\
\text { much? }\end{array}$ & $\begin{array}{l}\text { Quanto } \\
\text { custa? }\end{array}$ & Quanto custara a empresa? \\
\hline
\end{tabular}

Fonte: Adaptado pelo autor de Lima Junior e Freitas, 2005.

\subsection{Indicadores de Desempenho}

É comum que a gestão organizacional avalie seu desempenho apenas aplicando indicadores de natureza econômica/financeira, deixando de lado o acompanhamento do desempenho no que diz respeito a atividade de produção e operações que são fatores determinantes para a competitividade, assim uma condição básica para a realização de uma boa gestão é possuir um conjunto de indicadores que representem o desemprenho de sua organização a nível de eficiência produtiva (SILVA, 2013).

Para a identificação de produtividade de processos são adotados indicadores de desempenho, dos quais se faz presente o Overall Equipment Effectiveness (OEE), que Moellmann et.al, (2006) conceitua como um indicador responsável por traçar um comparativo entre os resultados reais e ideais para uma máquina ou processo.

O OEE é o produto de três elementos: disponibilidade, desempenho/performance e qualidade, e consiste na utilização de um conjunto de dados coletados e equações para obtenção de resultados numéricos (CHIARADIA, 2004).

Conforme Gambaro (2015), a disponibilidade, desempenho e qualidade podem ser calculados com base em equações que consideram as seis grandes perdas definidas por Nakajima (1989). 
Figura 1 - Representação das seis grandes perdas aplicadas aos cálculos dos índices.

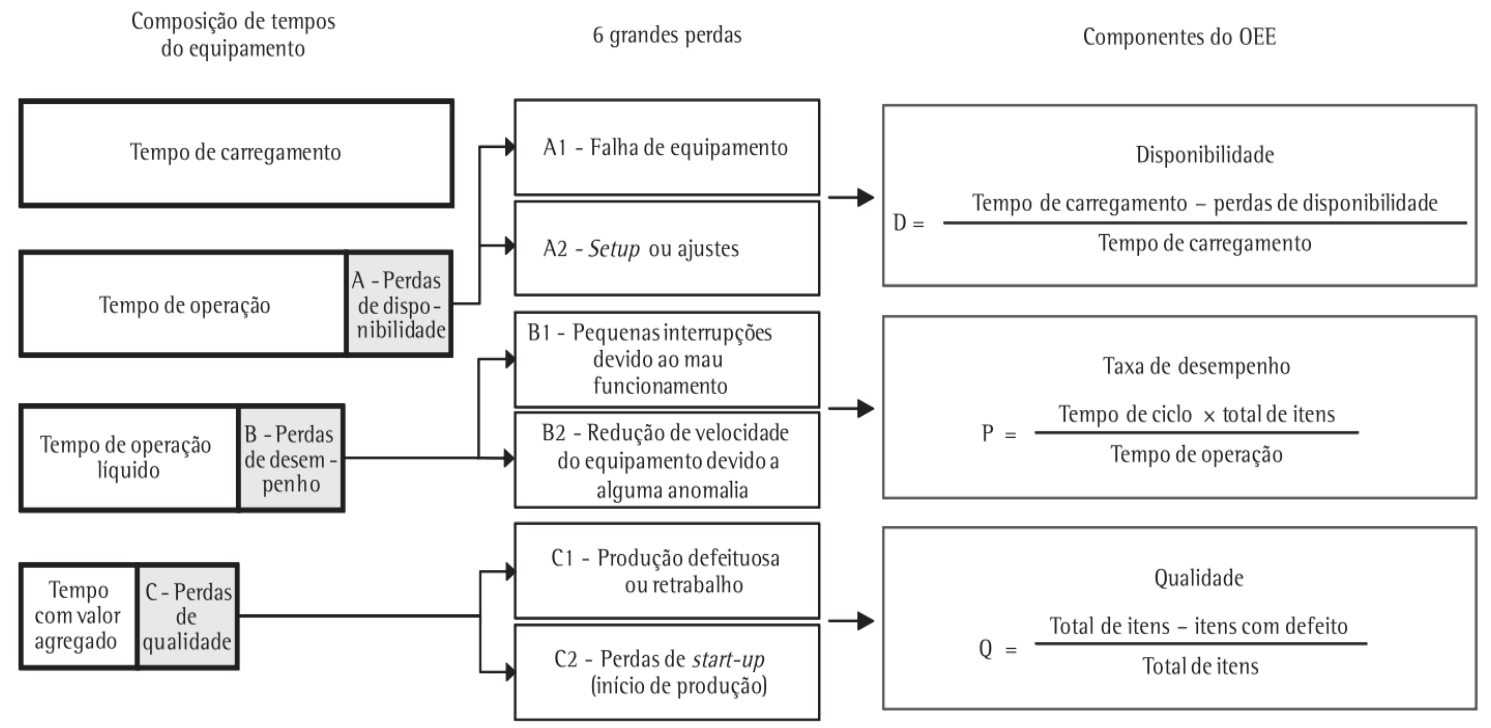

Fonte: Adaptado pelo autor de Busso e Miayke, 2015.

\section{$5 \quad$ Metodologia}

Foi desenvolvido no presente trabalho uma pesquisa exploratória, de abordagem qualiquantitava e de natureza aplicada, tratando-se de um estudo de caso que consistiu na aplicação de ferramentas, métodos e/ou modelos matemáticos/estatísticos, visando a identificação das causas/comportamentos que influenciam diretamente na conduta do ambiente estudado (NASCIMENTO, 2016).

A empresa na qual o presente trabalho foi ambientado trata-se de uma instituição de ensino superior do estado do Ceará, que contempla diversos cursos de graduação em diversas áreas. A mesma detém de uma de núcleos específicos de estudo/praticas, dos quais fazem parte ambientes como: Clinica de fisioterapia, Farmácia universitária e complexo odontológico nos quais se possui como foco o fomento do conhecimento teórico através da prática dos estudantes a partir do fornecimento de serviços à comunidade externa.

Após observar a rotina do complexo, adotou-se a metodologia de mapeamento e redesenho de processos presente no BPM CBOK (2013). Essas etapas são consideradas fundamentais para o desenvolvimento acertado do mapeamento de processos com base na metodologia BMPN.

O desenvolvimento e aplicação destas etapas seguiu um fluxo baseado em Stage-Gate, os estágios (etapas) a serem atingidos foram definidos da seguinte forma: (I) coleta de dados por observação; (II) coleta de dados sob a visão dos stakeholders; (III) comparação entre as duas 
perspectivas; (IV) Documentação do fluxograma simples e validação do processo real; (V) mapeamento do processo; (VI) acompanhamento do processo e documentação dos tempos, paradas e atividades; (VII) aplicação do OEE e ferramentas; (VIII) proposta de melhorias, Organizadas na figura abaixo.

Figura 2 - Representação das etapas dentro da metodologia do BPM CBOK.

\begin{tabular}{|c|c|c|}
\hline \multicolumn{2}{|c|}{ Etapas I, II e III } \\
\hline Estudo do processo atual & Definição dos objetivos & Identificação das variaveis \\
\hline Ducumentação do processo \\
\hline Etapas IV e V \\
\hline Enálise dos gargalos e fluxos \\
\hline Etapa VIII \\
\hline Proposta de melhoria \\
\hline
\end{tabular}

Fonte: Autor, 2019.

Para a análise de gargalos e fluxos, realizou-se aplicação de algumas das ferramentas da qualidade como auxílio na identificação das causas raízes dos gargalos, auxiliando ainda na composição do cenário futuro para aplicações de melhoria. O Quadro 4 representa a distribuição das ferramentas em função de suas aplicações.

Quadro 2 - Ferramentas Utilizadas.

\begin{tabular}{|l|l|}
\hline Ferramenta & Função \\
\hline OEE & Verificar produtividade do processo \\
\hline Pareto & Identificação do problema raiz \\
\hline Ishikawa & Identificação das causas do problema \\
\hline $5 \mathrm{~W} 2 \mathrm{H}$ & Plano de ação (melhorias) \\
\hline
\end{tabular}

Fonte: Autor, 2019. 
A fim de oferecer dados suficientes para aplicação dos indicadores de desempenho (OEE), foram realizados acompanhamentos presenciais com alunos de rendimento considerado médio, obtido através do cálculo da média, realizado com as notas obtidas pelos mesmos até o presente momento.

Quanto ao $O E E$, não foram encontradas na literatura aplicações deste índice para processos de serviço clínicos ou educacionais. No entanto, o processo estudado dispõe de equipamentos, bem como de um fluxo padrão de atividades e etapas. Assim tomando como base o OEE foram realizadas as seguintes adaptações a fim de solucionar essa deficiência. Como apresentado no quadro abaixo.

Quadro 3 - Equações

\begin{tabular}{|c|c|c|}
\hline Função & Função & Legenda \\
\hline $\begin{array}{l}\text { Tempo de } \\
\text { Operação }\end{array}$ & $\mathrm{TO}=\mathrm{TDD}-\left(\mathrm{TS}+\sum \mathrm{PNP}\right)$ & $\begin{array}{l}\text { TO }=\text { Tempo de operação } \\
\text { TS }=\text { Tempo de Setup }\end{array}$ \\
\hline $\begin{array}{c}\text { Índice de } \\
\text { Disponibilidade }\end{array}$ & $\mathrm{ID}=\frac{\mathrm{TO}}{\mathrm{TDD}}$ & $\begin{array}{l}\text { PNP }=\text { Paradas não programadas } \\
\text { TDD = Tempo total disponível para }\end{array}$ \\
\hline $\begin{array}{l}\text { Índice de } \\
\text { Eficiência }\end{array}$ & $\mathrm{IE}=\frac{\mathrm{TO}-\left(\sum \mathrm{PP}+\sum \mathrm{TV}\right)}{\mathrm{TO}}$ & $\begin{array}{l}\text { operação } \\
\text { TO = Tempo de operação }\end{array}$ \\
\hline $\begin{array}{c}\text { Índice Global de } \\
\text { Eficiência }\end{array}$ & $\mathrm{OEE}=(\mathrm{ID}) \mathrm{x}(\mathrm{IE}) \mathrm{x}(\mathrm{IQ})$ & $\begin{array}{l}\text { TO = Tempo de operação } \\
\text { PP = Paradas programadas } \\
\text { TV }=\text { Tempo de validação }\end{array}$ \\
\hline
\end{tabular}

Fonte: Autor, 2019.

\section{Estudo de Caso}

De acordo com Tiedmann (2005), o objetivo do atendimento clínico universitário consiste em suprir as necessidades do aperfeiçoamento técnico e prático da formação do cirurgião dentista, atendendo os ideais éticos, humanos e profissionais e suprindo as necessidades dos usuários desse serviço, conforme as diretrizes institucionais.

Assim, por possuir o enfoque em uma atividade educacional, o processo essencial de uma clínica pertencente a uma instituição de ensino superior (IES) apresenta dois objetivos distintos, 
porém complementares. $O$ objetivo principal consiste na capacitação profissional, caracterizando assim o aprendizado como produto principal e função de sua existência. Enquanto a execução dos procedimentos visa ofertar ao paciente um atendimento gratuito e de qualidade, definindo assim um objetivo secundário, mas não menos importante quanto o objetivo principal.

Diante das informações supracitadas e atendendo os objetivos deste trabalho, respeitando a sequencia de atividades propostas, como o mapeamento e documentação do processo atual (Anexos A e B), as atividades que não agregavam valor ou que não condiziam com seus objetivos foram consideradas desnecessárias para o processo. O Quadro 4 apresenta essas atividades e o tempo gasto em média para realiza-las.

Quadro 4 - Atividades.

\begin{tabular}{|c|c|c|}
\hline Atividade & Tipo de atividade & Tempo médio identificado \\
\hline Diagnóstico & Processo de suporte & 2 horas e 10 min \\
\hline Raio $\mathrm{x}$ & Processo suporte & 18 minutos \\
\hline
\end{tabular}

Fonte: Autor, 2018.

Afim de complementar os dados levantados e apresentados no quadro anterior, foram aplicados os indicadores de desempenho e utilizando as equações descritas na metodologia, os dados foram organizados na Tabela 1, para melhor compreensão da aplicação do OEE.

Tabela 1 - Tempos do processo.

\begin{tabular}{|c|c|c|c|c|c|c|}
\hline ESTUDO & TO & TV & TS & PNP & $\mathbf{P P}$ & TDD \\
\hline $1^{\circ}$ & 126,46 & 29,31 & 15,32 & 78,22 & 12,34 & \multirow{4}{*}{220} \\
\hline $2^{\circ}$ & 136,76 & 23,32 & 23,76 & 59,48 & 15,20 & \\
\hline $3^{\circ}$ & 157,75 & 19,09 & 13,05 & 49,2 & 11,43 & \\
\hline $4^{\circ}$ & 136,25 & 24,06 & 18,67 & 65,08 & 16,20 & \\
\hline
\end{tabular}

Fonte: Autor, 2019. 
Com base nos tempos obtidos e apresentados na Tabela 1, foram realizados os cálculos para os Índices de Disponibilidade (ID) equação 2, de Eficiência (IE) equação 3, e o $O E E$ equação 4, para o índice de qualidade (IQ) foi atribuído o valor de 1, uma vez por se tratar de um ambiente educacional, a realização dos retrabalhos é algo intrínseco do processo. A figura 2, a seguir representa os resultados das aplicações das equações dos indicadores de desempenho

Figura 2 - Gráfico com indicadores de desempenho

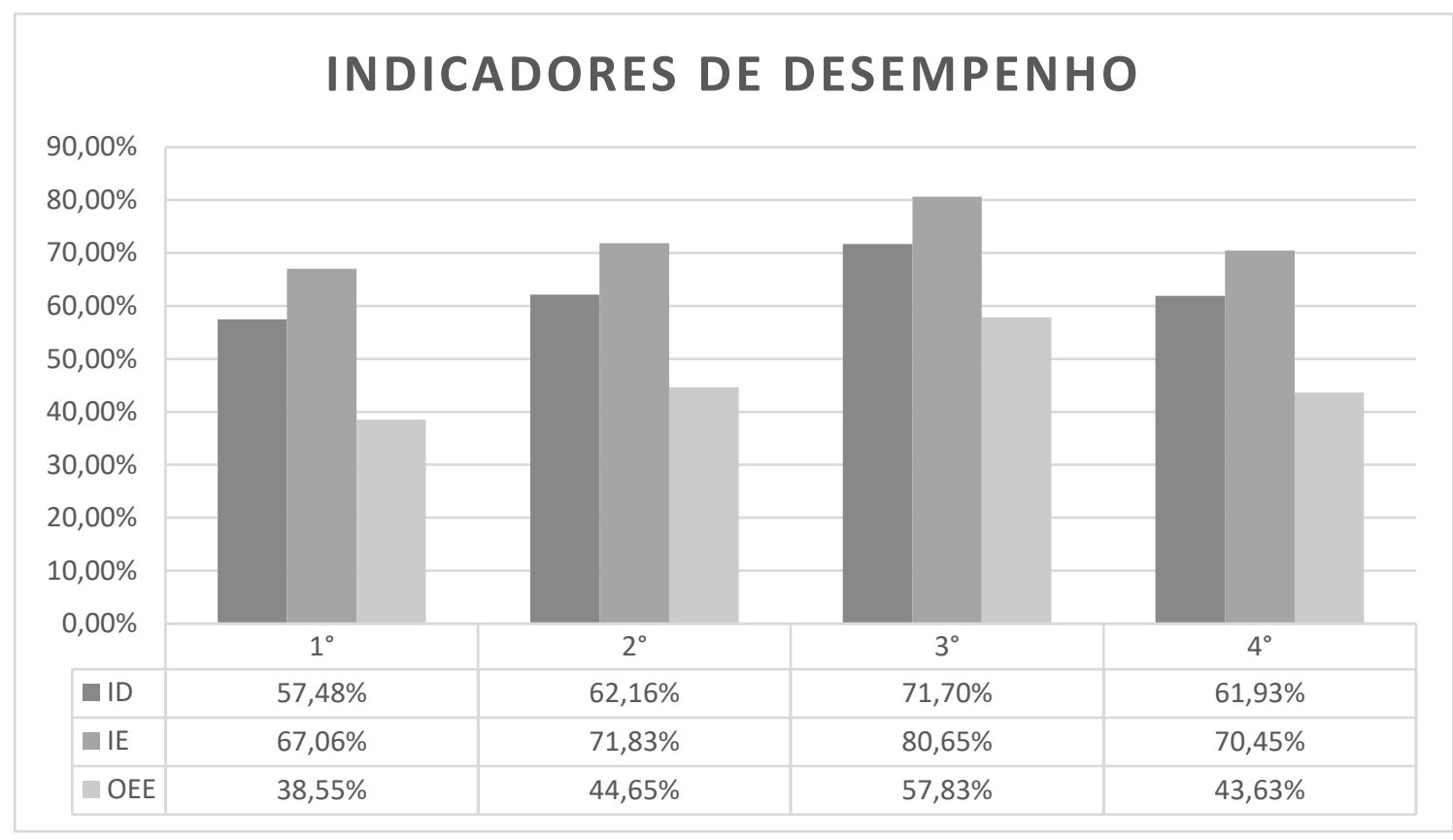

Fonte: Autor, 2019.

Além das atividades que não agregavam valor ao processo específico, alguns outros gargalos foram identificados. Os mesmos correspondiam às dificuldades na realização das tarefas, seja por fatores internos ou externos ao processo. Como exemplo de atividades dispensáveis ao processo, pode-se citar a ausência de padronização na definição das etapas do atendimento. Essa ausência de padronização, gera gargalos e influência diretamente na percepção dos índices de produtividade, deixando-os abaixo do esperado. Esses gargalos foram organizados em um diagrama de Pareto, com finalidade de classificar o nível de urgência dos gargalos a serem resolvidos. A figura 3 representa esse diagrama. 
Figura 3 - Gráfico de Pareto.

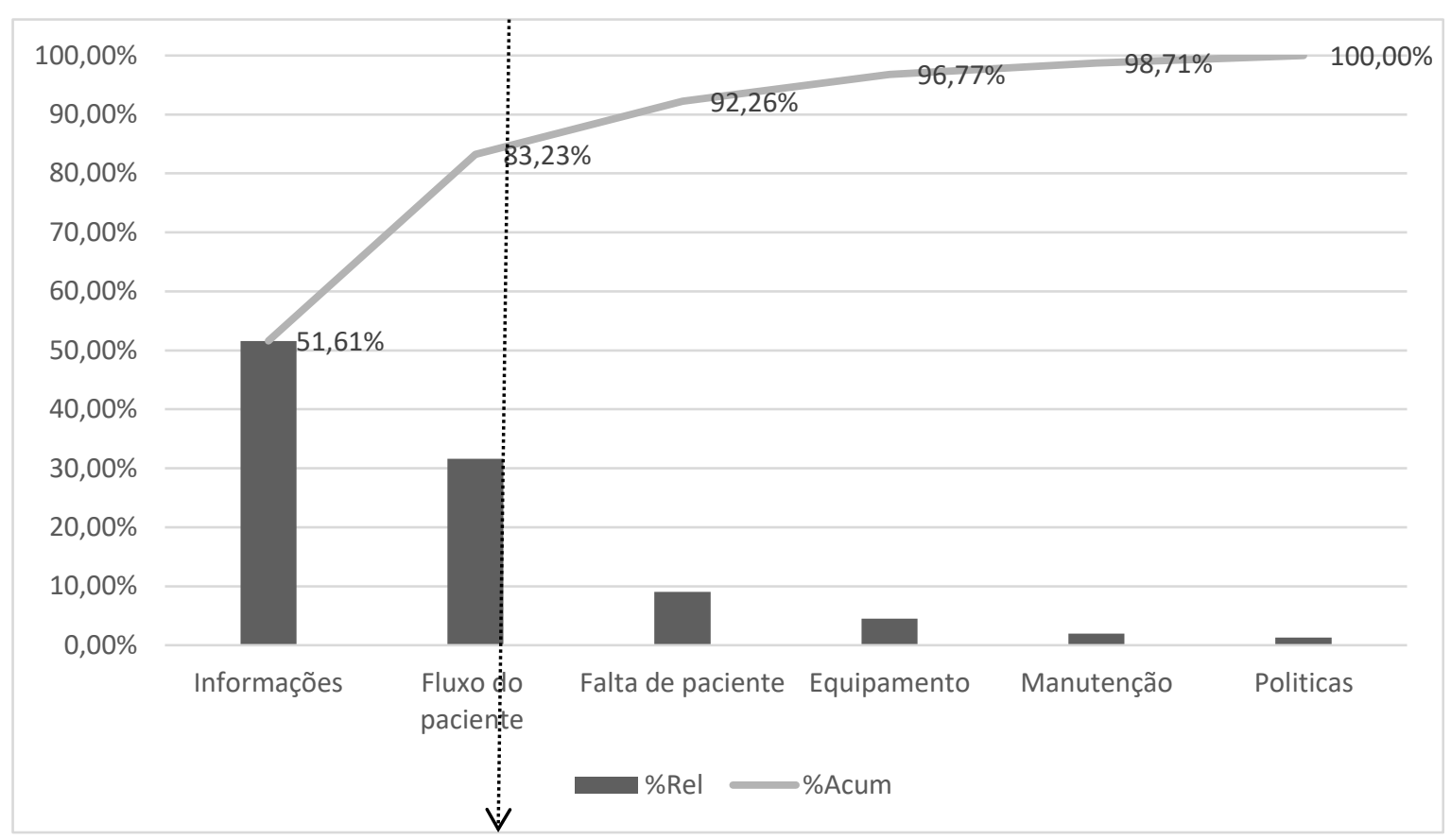

Fonte: Autor, 2019.

Com a aplicação do gráfico de Pareto percebe-se que $80 \%$ dos problemas estão centralizados nas duas primeiras causas que e com isso identificou-se que o erro mais recorrente no processo se dá devido a falha no fluxo de informações, o que provoca uma confusão no fluxo de pacientes. Com isso as clínicas mais avançadas atendem toda uma demanda desnecessária de clientes, o que provoca ausência de pessoas a serem atendidas nas clínicas iniciais. Objetivando traçar um plano de melhoria foi aplicado o diagrama de Ishikawa (Figura 4) com o objetivo de encontrar as causas raízes para essa falha no fluxo informacional e direcionar as ações de melhoria a mesma. 
Figura 4 - Diagrama de causa e efeito

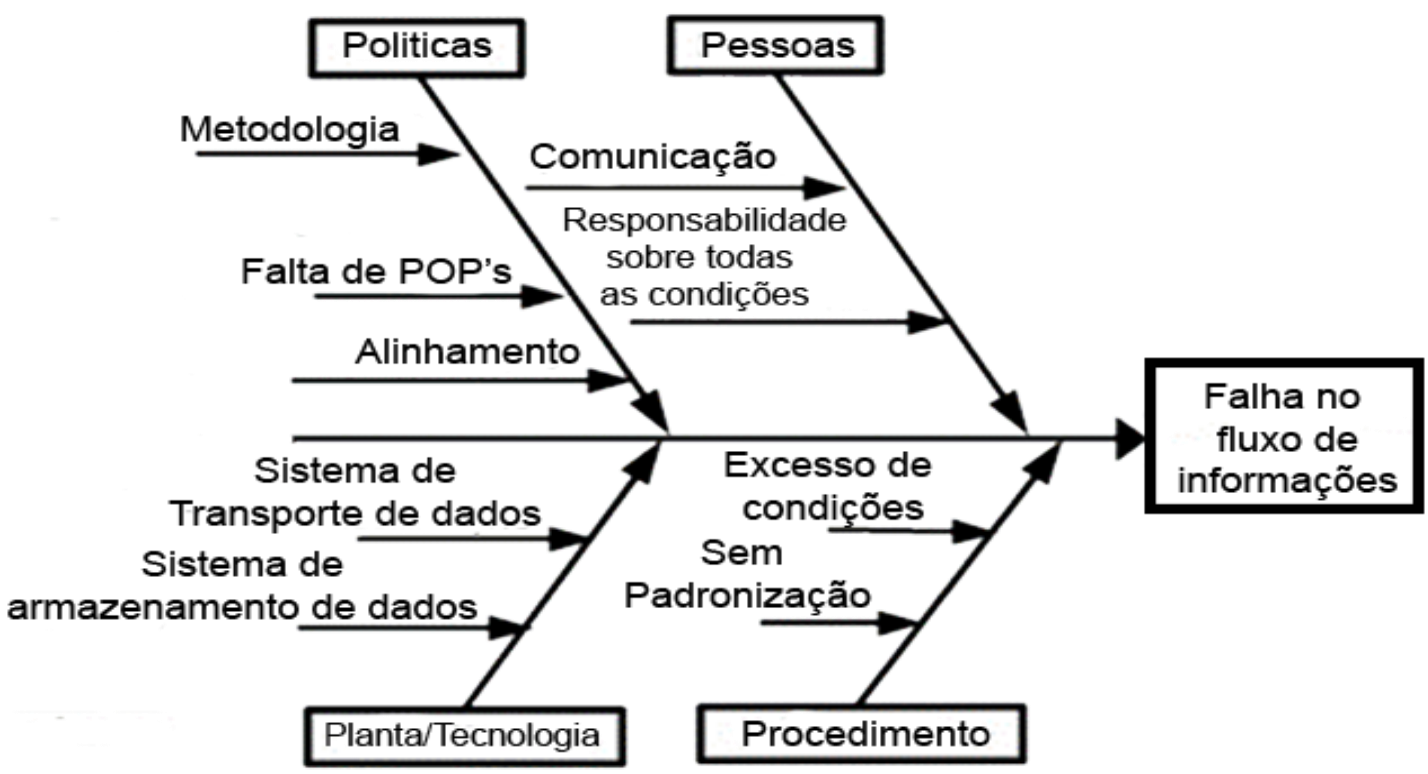

Fonte: Autor, 2019.

De acordo com O BPM CBOK (2013), o fluxo de dados é apresentado como fator primordial para o bom funcionamento do processo, uma vez que falhas na comunicação impossibilitam a execução de forma conjunta e precisa das atividades relacionadas diretamente com o fluxo do processo. $\mathrm{O}$ mesmo manual afirma que análise do processo deve ser concisa e apresentar todas as informações de forma clara, sem que sejam mascaradas as necessidades de melhoria. Isso porque, busca-se garantir uma real representação e direcionar corretamente as ações de melhoria.

Ao fim da aplicação das ferramentas, identificou-se que a maior falha no andamento do processo estudado é proveniente do sistema ineficiente de troca de informações, fazendo com que o fluxo das atividades não possua consistência. Ocasionando assim os demais gargalos citados ao longo desse trabalho. O diagrama de Ishikawa utilizado, fundamenta-se nos "4P's": Politicas, Pessoas, Planta e Procedimento. As falhas encontradas foram linkadas a cada um dos "P's" afim de identificar as causas raízes do problema e direcionar as ações de correção (Anexo C).

\section{$7 \quad$ Considerações Finais}

O presente trabalho conciliou algumas ferramentas da qualidade com as etapas do guia de mapeamento de processos e apresentou ao fim do seu desenvolvimento dados concisos que devem servir de auxilio e justificativa para a necessidade da implementação de uma gestão 
baseada em processos. Se aplicado, deve gerar ganhos de desempenho significativos para o processo, assim como para o complexo de forma geral.

Referências

ASSOCIATION OF BUSINESS PROCESS MANAGEMENT PROFESSIONALS - ABPMP. Guia para gerenciamento de processos de negócio: corpo comum de conhecimento. V 3.0. Brasília: ABPMP, 2013.

BARRETO, Juliana; LOPES, Luis Felipe dias. Análise de falhas no processo logístico devido à falta de um controle de qualidade. Revista Produção Online, v. 5, n. 2, 2014.

BROCKE, Jan Vom; Rosemann. Manual de BPM: gestão de processos de negócios. Porto Alegre: Bookman, 2013. $384 \mathrm{p}$.

CARVALHO, M. M. et al. Gestão da qualidade: teoria e casos. 6. ed. Rio de Janeiro: Elsevier, 2005.

CHIARADIA, Áureo José Pillmann. Utilização do indicador de eficiência global de equipamentos na gestão e melhoria contínua dos equipamentos: um estudo de caso na indústria automobilística. 2004. $133 \mathrm{f}$. Dissertação (Mestrado) - Escola de Engenharia, Universidade Federal do Rio Grande do Sul, Porto Alegre/RS, 2004.

CRUZ, Tadeu. Manual para gerenciamento de processos de negócio: metodologia DOMP. São Paulo: Atlas S.A, 2015.

GAMBARO, Alex Augusto. OEE (Eficiência Global dos Equipamentos) como uma ferramenta valiosa para o monitoramento da eficiência produtiva em empresa de pequeno porte. 2015. 17 f. Monografia (Graduação) - Universidade Federal do Paraná, Curitiba/PR, 2015.

KOSLOSKI, R. A. D.; CALAZANS, A. T. S.; GUIMARÃES, F. de A. Proposta de modelo de medições para contratação do gerenciamento de processos de negócio (BPM - Business Process Management). Journal of Information Systems and Technology Management, v. 13, n. 2, p. 275-300, 2017.

LIMA JUNIOR, Oscar Pereira de; FREITAS, Adolfo Júlio Porto de. Estudo das disfunções do fluxo de informação do arquivo do departamento financeiro da empresa Z. S/A: Aplicação da técnica 5W2H. Biblionline, v. 1, n. 1, 2005.

LINS, Bernardo F. E. Ferramentas básicas da qualidade. Ciência da Informação, v. 22, n. 2, 1993.

LIRA, Manoel Fabiano Leite. et al. Gestão por Indicadores como Prática Lean para Melhoria Contínua : Um Estudo de Caso em um Produtor de Imunobiológicos. Journal of Lean Systems, v. 1, p. 23-38, 2016.

MEIRELES, Manuel. Ferramentas administrativas para identificar observar e analisar problemas. São Paulo: Arte \& Ciência, 2001. 
MOELLMANN, Artur Henrique et al. Aplicação da teoria das restrições e do indicador de eficiência global do equipamento para melhoria de produtividade em uma linha de fabricação. Revista Gestão Industrial, v. 2, n. 1, 2006.

NAKAJIMA, S. Introdução ao TPM. São Paulo: IMC Internacional Sistemas Educativos, 1989.

NASCIMENTO, Francisco Paulo do. Classificação da Pesquisa: objetivos e procedimentos. In:

Metodologia da pesquisa científica: teoria e prática - como elaborar TCC. Brasília: Thesaurus, 2016. p. 1-11.

OLIVEIRA, Djalma de Pinho Rebouças. Administração de processos: conceitos, metodologias, práticas. 5. ed. São Paulo: Atlas S.A, 2013.

PAIM, Rafael et al. Gestão de processos: pensar, agir e aprender. Porto Alegre: Bookman, 2009. 320 p. v. 1.

PRADELLA, Simone; FURTADO, João Carlos; KIPPER, Liane Mählmann. Gestão de processos: da teoria à prática. São Paulo: Atlas, 2012. 141 p.

SHEWHART, Walter Andrew; DEMING, William Edwards. Método estatístico do ponto de vista do controle de qualidade. [S.1.]: Courier Corporation, 1986.

SILVA, Jéssica Sousa. O mapeamento de processos organizacionais no setor público: estudo de caso do escritório de processos da Agência Nacional de Vigilância Sanitária - Anvisa. 2014. 58 f. Monografia (Bacharelado em Gestão de Políticas Públicas) - Faculdade de Economia, Administração e Contabilidade, Universidade de Brasília, Brasília/DF, 2014.

SLACK, Nigel et al. Gerenciamento de operações e processos. Porto Alegre: Bookman, 2013.

TIEDMANN, Claus Robert; LINHARES, Eleana; SILVEIRA, João Luiz Gurgel Calvet da. Clínica integrada odontológica: perfil e expectativas dos usuários e alunos. Pesquisa Brasileira em Odontopediatria e Clínica Integrada, v. 5, n. 1, 2005. 
ANEXOS

ANEXO A- PROCESSO DE ANAMNESE (SUPORTE)

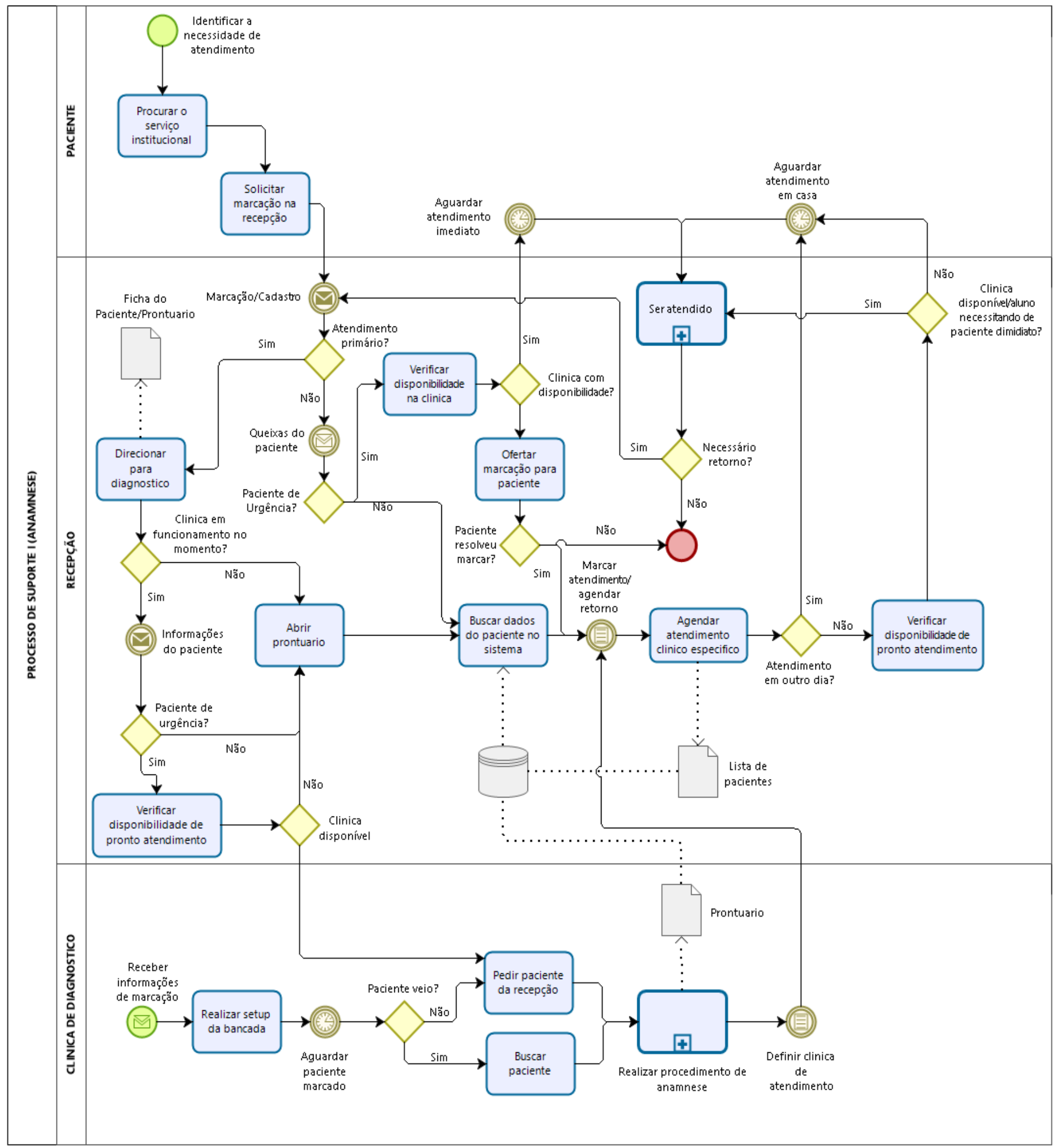

Fonte: Autor, 2019. 
ANEXO B - PROCESSO CLINICA I (ESSENCIAL)

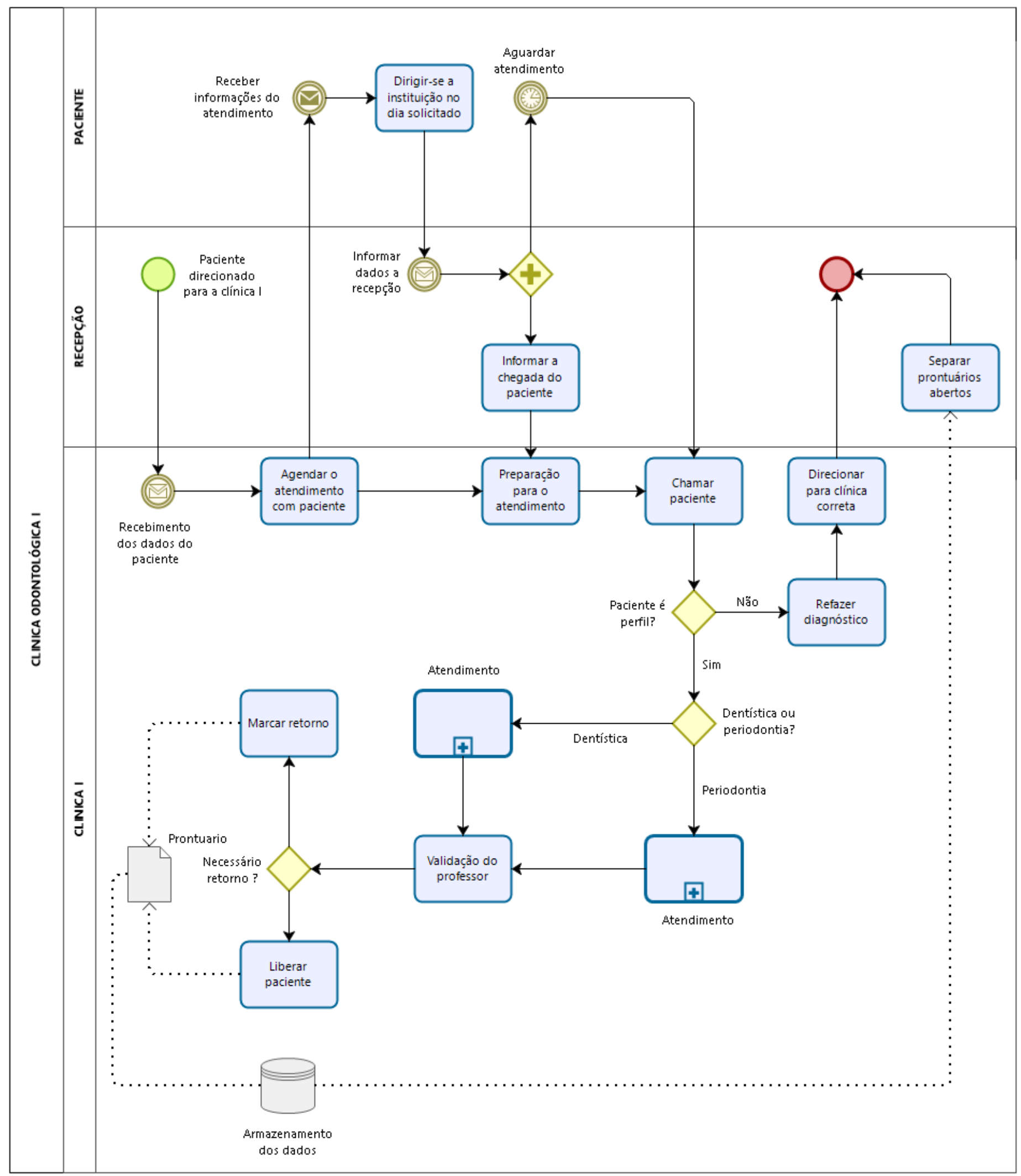

Fonte: Autor, 2019. 
ANEXO C

Plano de melhorias (Clínica Odontológica I)

\begin{tabular}{|c|c|c|c|c|c|}
\hline \multicolumn{4}{|c|}{ Elaborado por: Diego Silva Mota } & \multicolumn{2}{|l|}{ Revisado por: Jair Paulino } \\
\hline \multicolumn{2}{|l|}{ Versão: 2.0} & \multicolumn{2}{|l|}{ Data: $22 / 05 / 2019$} & & \\
\hline \multicolumn{5}{|c|}{$4 W$} & $1 \mathrm{H}$ \\
\hline O que? (What?) & Porquê? (Why?) & Onde? (Where?) & Quem? (Who?) & Quando? (When?) & Como? (How?) \\
\hline $\begin{array}{c}\text { Redesenho do } \\
\text { processo da clínica I }\end{array}$ & $\begin{array}{l}\text { Melhoria do fluxo de atividades } \\
\text { internas, padronização dos } \\
\text { procedimentos, tornar auxiliar a } \\
\text { tomada de decisões }\end{array}$ & $\begin{array}{l}\text { Disciplina de clínica } \\
\text { I }\end{array}$ & $\begin{array}{l}\text { Engenharia de } \\
\text { Produção }\end{array}$ & Até dezembro de 2019 & $\begin{array}{c}\text { Através do mapeamento } \\
\text { atual }\end{array}$ \\
\hline $\begin{array}{c}\text { Definição de } \\
\text { procedimentos } \\
\text { operacionais padrão }\end{array}$ & $\begin{array}{l}\text { Padronizar as atividades para que } \\
\text { não haja demora excessiva e } \\
\text { reduzindo a incerteza do processo }\end{array}$ & $\begin{array}{l}\text { Disciplina de clínica } \\
\text { I }\end{array}$ & $\begin{array}{l}\text { Engenharia de } \\
\text { Produção }\end{array}$ & Até 2020 & $\begin{array}{c}\text { Desenvolvendo documentos } \\
\text { que garantam a execução } \\
\text { padronizada das atividades }\end{array}$ \\
\hline $\begin{array}{l}\text { Fluxograma do } \\
\text { paciente }\end{array}$ & $\begin{array}{l}\text { Garantir que o paciente seja } \\
\text { direcionado corretamente }\end{array}$ & $\begin{array}{c}\text { Complexo } \\
\text { odontológico }\end{array}$ & $\begin{array}{l}\text { Engenharia de } \\
\text { Produção + } \\
\text { Professores do } \\
\text { Curso de } \\
\text { Odontologia }\end{array}$ & Até dezembro de 2019 & $\begin{array}{l}\text { Definindo um fluxograma } \\
\text { padrão para todo o processo }\end{array}$ \\
\hline $\begin{array}{c}\text { Implementação do } \\
\text { sistema já } \\
\text { desenvolvido } \\
\text { (SisOdonto) }\end{array}$ & $\begin{array}{c}\text { Facilitar e garantir a consistência } \\
\text { nas informações trocadas entre os } \\
\text { setores }\end{array}$ & $\begin{array}{c}\text { Complexo } \\
\text { odontológico }\end{array}$ & CTI & Até 2021 & $\begin{array}{l}\text { Utilizando equipamentos já } \\
\text { disponíveis e subutilizados }\end{array}$ \\
\hline $\begin{array}{c}\text { Programa de } \\
\text { alinhamento entre } \\
\text { colaboradores e } \\
\text { estratégias } \\
\text { organizacionais }\end{array}$ & $\begin{array}{c}\text { Garantir que todos os } \\
\text { colaboradores entendam e } \\
\text { participem de todos os objetivos e } \\
\text { metas traçadas pela gestão }\end{array}$ & $\begin{array}{c}\text { Complexo } \\
\text { odontológico }\end{array}$ & $\begin{array}{l}\text { Coordenação do } \\
\text { curso de } \\
\text { odontologia }\end{array}$ & Até outubro de 2019 & $\begin{array}{c}\text { Reuniões. Palestras, Manual } \\
\text { do colaborador }\end{array}$ \\
\hline
\end{tabular}

Fonte: Autor, 2019. 